common analysis in systems neuroscience is to estimate the firing rate of a neuron by averaging the response across many stimulus repetitions. This analysis is appropriate if the underlying network supports both a time-evolving rate (which is effectively signal and is recovered by averaging) and spiking variability (which is effectively noise and is suppressed by averaging). Furthermore, experimentalists often attempt to segregate response variability into firing-rate variability and spiking noise $\mathrm{e}^{1,2}$. It is generally assumed that the former can influence behavior ${ }^{14}$ or perception ${ }^{15}$, whereas the latter effectively acts as measurement noise. These are indeed fair assumptions for a network of the class simulated by Litwin-Kumar and Doiron ${ }^{3}$. Firing-rate fluctuations are shared among neurons (and can affect behavior), whereas spiking variability is local in time and mostly uncorrelated between neurons ${ }^{4}$ (and would have difficulty affecting behavior).
A number of outstanding questions remain. Can the proposed class of spiking network instantiate the full range of dynamics that can be built from rate-based units, including dynamics that involve a continuous evolution of the rate (for example, integrators, limit cycles and chaotic attractors)? If so, should our models and hypotheses dispense with the nuisance of spiking variability and simulate dynamics directly at the level of rates? Or might structured details of spiking influence the evolution of the network at the level of rates, as has often been suggested? Such questions underscore that our field is not yet completely certain which aspects of a spike train are signal and which are noise. Still, we seem to have come to a fairly solid understanding that not every spike is sacred and that the dynamics of many networks are best described at the abstract level of rates.

COMPETING FINANCIAL INTERESTS

The authors declare no competing financial interests.
1. Churchland, A.K. et al. Neuron 69, 818-831 (2011).

2. Churchland, M.M. et al. Nat. Neurosci. 13, 369-378 (2010).

3. Litwin-Kumar, A. \& Doiron, B. Nat. Neurosci. 15 1498-1505 (2012)

4. Renart, A. et al. Science 327, 587-590 (2010).

5. Sompolinsky, H., Crisanti, A. \& Sommers, H.J. Phys. Rev. Lett. 61, 259-262 (1988).

6. Shadlen, M.N. \& Newsome, W.T. Curr. Opin. Neurobiol. 4, 569-579 (1994).

7. van Vreeswijk, C. \& Sompolinsky, H. Science 274 1724-1726 (1996).

8. Vogels, T.P. \& Abbott, L.F. J. Neurosci. 25 10786-10795 (2005).

9. Troyer, T.W. \& Miller, K.D. Neural Comput. 9, 971-983 (1997).

10. Carandini, M. PLoS Biol. 2, e264 (2004).

11. London, M., Roth, A., Beeren, L., Hausser, M. \& Latham, P.E. Nature 466, 123-127 (2010).

12. Rajan, K., Abbott, L. \& Sompolinsky, H. Phys. Rev. E 82, (2010).

13. Deco, G. \& Hugues, E. PLOS Comput. Biol. 8 e1002395 (2012).

14. Churchland, M.M., Afshar, A. \& Shenoy, K.V. Neuron 52, 1085-1096 (2006).

15. Britten, K.H., Newsome, W.T., Shadlen, M.N., Celebrini, S. \& Movshon, J.A. Vis. Neurosci. 13 87-100 (1996).

\title{
Cdk5 keeps memory on Trk
}

The structural and functional plasticity of synaptic connections is thought to contribute to the formation of new memories in the brain. There are a number of molecules that participate in the establishment of synaptic plasticity and in memory formation, including brain-derived neurotrophic factor (BDNF). Its receptor, $\operatorname{TrkB}$, is a receptor tyrosine kinase that can, when activated, trigger autophosphorylation of its tyrosine residues. However, several other kinases can also phosphorylate this receptor on serine residues, and whether these forms of phosphorylation are actually critical for synaptic plasticity and memory remains an open question. On page 1506 of this issue, Lai and colleagues provide some important answers to this question by looking specifically at the role of the proline-directed serine and threonine kinase Cdk5 in the activation of TrkB and the subsequent effects on synaptic plasticity and memory.

The authors previously found that Cdk5 can phosphorylate TrkB on serine 478 to promote dendritic growth. To further determine the molecular mechanisms acting downstream of this signaling branch of the TrkB receptor and uncover the physiological relevance of S478 phosphorylation by $\mathrm{Cdk} 5$, Lai and colleagues generated a knock-in mouse in which TrkB was swapped with a phosphorylation-deficient S478A mutant TrkB receptor. They found that TrkB phosphorylated on its serine 478 colocalizes with

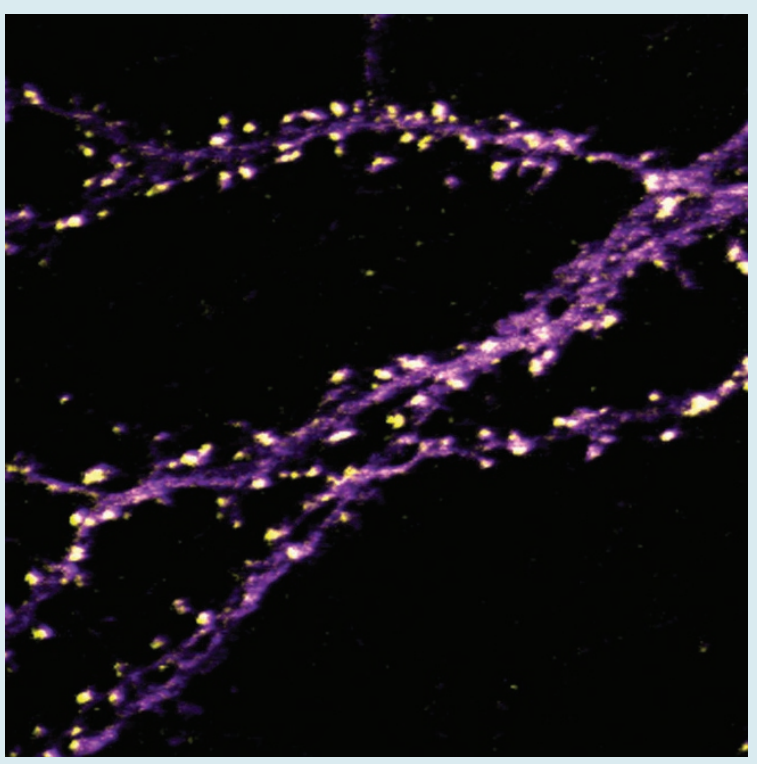
PSD-95 in dendritic spines in hippocampal neurons in cultures (see image)

and that this phosphorylation is crucial for BDNF-induced spine morphogenesis and glutamate-induced spine growth. Furthermore, S478 phosphorylation promoted the interaction of TrkB with the Rac-GEF TIAM1 and the activation of the Rac-PAK pathway, thereby linking phosphoS478-dependent signaling to the machinery that modulates actin dynamics and mediates spine remodeling. Crucially, S478 phosphorylation impaired CA3-CA1 long-term potentiation in hippocampal slices and S478A mutant mice exhibited severe deficits in spatial memory and novel object recognition tasks. However, their ability to learn contextual fear was intact.

The study by Lai and colleagues provides some new insights into to how Cdk5 and BDNF contribute to synaptic plasticity, learning and memory, and presents compelling evidence of the importance of serine phosphorylation of a receptor tyrosine kinase in this process. 